\title{
Behavior analysis of factors affecting safety management to reduce incidents in the pelletizing industry using the system dynamics approach
}

\author{
Hajimoradi, A., Zare Mehrjerdi*, Y. and Hatamzade, S. \\ Department of Industrial Engineering, Faculty of Industrial Engineering, Yazd University, Yazd, Iran \\ *Corresponding author email: mehrjerdyazd@gmail.com
}

\begin{abstract}
The purpose of this study was to analyze the behavior of effective factors on safety management to reduce incidents in the pelletizing industry using the system dynamics approach. Risk assessment for the Sechahon pelletizing plant was performed using the FMEA method. A total of 625 risks were identified in this complex of which 286 are high risk RPNs and need to be scrutinized. These risks were categorized according to their nature and consequences in five physical categories, exposure to dust, ergonomics, emergencies and psychosocial risks, so that they can be reviewed in detail. The results showed that decreasing the number of incidents in the risk reduction scenario would reduce the incidence earlier, and is initially more than other policies. The scenario of risk reduction is the best scenario in the short term. The technology improvement scenario shows an incremental growth trend, but the scenario of the current situation can better decrease the number of incidents. The human resource management and safety management scenario will initially be on an upward trend and will continue to decline. The human resource management and safety management scenario is a good way to reduce disasters in the long run.
\end{abstract}

Keywords: safety management, system dynamics, incident reduction, pelletizing industry.

DOI: $10.7176 / \mathrm{IEL} / 10-1-06$

Publication date: February $29^{\text {th }} 2020$

\section{Introduction}

Millions of work-related incidents occur every year. Some of these events cause death, and others cause temporary disability, which may last for months. Workers are often exposed to various hazards in industrial environments despite many machines and tools. With the advent of technology and the increased use of machinery in production, the risks and incidence of incidents in these environments will increase. The occurrence of incidents damages the device and equipment of raw materials, products or work environment or associates immeasurable physical or mental harm. Therefore, it is important to take precautionary measures and to pay attention to safety management in industrial projects to prevent or reduce occupational incidents. Incidents and occupational diseases are considered to be important barriers to productivity and production. Among the factors of workplace incidence, the human error factor accounted for the largest share, i.e. 70 to $90 \%$ of the causes of the incident, according to studies. Insecure behavior is beyond the standard and defined limits of the system and can affect the level of safety and health of the system. Some of the high-risk behaviors in the workplace that endanger the health of the employees are poor physical condition at work (such as inappropriate sitting, lifting objects heavier than the standard set, kneeling in the long run), not using required respiratory, fatigue, and violence protection tools in the workplace. Also, these behaviors include non-observance of work instructions, the inappropriate use of personal protective 
equipment, the use of non-essential tools for doing work, and dealing with non-duties (Suri et al., 2011). The statistics of work-related incidents indicate that industrial incidents are not in Iran isolated from most developing countries (Kayjordi, 2011).

Today, the diversity and risk of workplace hazards are so high that in practice it is impossible to compensate for the consequences. For this reason, safety science has taken a completely preventive approach. One of the most prominent features of this science is its systematic functioning, which emphasizes the identification, evaluation, and control of all elements involved in all stages. The main elements of this system include humans, equipment, materials, and the environment. Among the elements mentioned above, human is the most critical factor, since this element, while dominating other elements, is capable of influencing three other elements by unsafe behaviors. The human factor in the immune system includes a wide range of people from senior management to simple workers (Rauf and Moezeni, 2017). Safety at work is a complex phenomenon and the issue of safety attitude and safety performance in industrial projects and even more complicated. In a capital-based society, it is commonplace for industry stakeholders, especially those at the bottom of the supply chain, to focus exclusively on completing projects in order to achieve the required quality standards with minimal time and cost. The lack of incentives to strengthen the safety culture at both organizational and project levels generally results in a weakness in safety history. Safety management improves performance indicators in workshops and industrial projects, so having an approach to safety management and safety management systems will enhance safety in industrial projects (Taqinejad, 2017).

Among the obvious capabilities of the concept of safety, there is sufficient capacity to identify cause-and-effect relationships and to design practical management and control approaches. These actions have come to the fore in the form of practical methods of safety management since the beginning of the development. As a result, safety skills in all aspects of the industry have been rising over the years, and there has been an increasing change in the growing awareness and ability to meet the required tools and techniques for controlling risks. Evidence of this issue is seen in many related regulations, standards, and laws. This process may seem normal and conceivable; nevertheless, practical experiences and results remind us incidents result in injuries and damage continues to occur despite the awareness of the causes and the existence of many proposed control tools (Taqinejad, 2017).

\section{Review of literature and research background}

In the past, the incidents included falling from a tree or a height, being hit and injured by domestic or wild animals, poisoning with plant material or animal poison and so on, but today, given the remarkable advances in industrial affairs and the possibility of using modern means of travel, the use of electric power and machinery, contact with chemicals, etc., human beings are facing countless incidents.

Incident: An incident can be defined as an unplanned event or incident. The incident is unexpected and unpredictable. If we want to open this issue further, the incident is the result of an unwanted event. In addition to the general definitions for the various incidents referred to, the definition of the work-related incident can be found in the article 60 of the Labor and Social Security Act (Dousti, 2014): Occupational incidents are events that occur during the performance of the task and, therefore, for the insured. The definition of an incident in the International Encyclopedia of Work is an unforeseen and unexpected event that causes injury and harm (Dousti, 2014). According to the standard definition of OHSAS 18001-2007, an incident is an event that results in injury, illness, 
or death

The significance of work-related incidents: Every year, tens of millions of workers are victims of the world, resulting in numerous deaths or disability.

A) Humanly

Any work-related incident, even partly, causes the worker and his or her family to be distressed.

B) Socially

Since the advancement of each social depends on the workforce of the community, the product of the work of each worker is not only the livelihood of his life and his family, but also the capital and backing of the economy of a society.

As we know, nearly 50 to $60 \%$ of people in each social group are people of working age.

C) Economically

Incidents, in any mode and degree, are economic losses for the worker, the employer, and the community. These losses are directly and indirectly. Direct losses include losses resulting from work interruptions due to incidents, medical expenses, and eventually damages for temporary, permanent incapacity or death. In calculating indirect losses, which are more than direct losses in all countries, losses caused by interruptions in the work of other workers due to helping the injured person, discussing the cause of the incident, disrupting the work order after the transfer of the worker to the hospital until the appointing the right person to do business, the damage to the machinery and, eventually, the damage resulting from the reduction of the work of the injured worker after returning to work (in the case of a disability) should be considered.

Management on the process of incidents: Management of the incident process, i.e. the investigation of the causes of incidents during work and preventive measures. Based on the proper management of incidents, it is possible to apply methods that minimize the economic costs of incidents. Managing the process of incidents involves examining the causes of incidents and taking preventive measures. The work-related incident is a fact that occurs during the work and there was no previous intention or planning for it.

Causes of work-related incidents: The performed studies indicate that in general, the work-related incident does not have a single cause, and the effect is due to technical and human causes. These causes depend on the type of work, the environment, the conditions of the work and the tools used and can be divided into two categories of direct and indirect causes:

\section{A. Direct causes}

The purpose of direct causes is the source of the main contributor to the occurrence of the incident. Given the work and industry, these can be summarized as follows: Moving goods, working with machinery, falling objects, dropping a worker from a height, improper use of tools, falling due to slipping, collision with a barrier, burn, as well as an incident with the vehicle in the workshop environment or when going back to the work place.

\section{B. Indirect causes}

These causes do not directly cause the incident, but if there are direct causes, they increase the chance of an incident. This group includes all the factors that cause fatigue, discomfort, and labor dissatisfaction. The most important of these are improper light, excessive sound, poor ventilation, inappropriate working temperature, long working hours, over-speeding, and other factors such as: Family, financial, relations with the employer and the supervisor, and so on. It should also be noted that along with these two groups of causes, lack of experience and 
work skills an $\mathrm{d}$ non-observance of the principles of immunity are important in causing work-related incidents.

\section{Research Literature}

Amin Fathi Biranvand (2017) used the research system dynamic approach to provide appropriate and optimal ways to investigate the causes of incidents and incidents by using time management, cost, and quality method. In this research, the required data were collected using surveying studies. In this research, the required data were collected using field studies using statistical methods and appropriate statistical tests. Then, the dynamic model of the study was plotted and simulated using Vesnim software. Finally, an appropriate strategy for managing time, cost, and quality was presented in the study of the causes of incidents and construction incidents, and then, the issues that led to the reduction of incidents in the construction workshops were expressed. Rauf and Moazeni (2017) in a paper entitled, "Managing the safety of contractors in the control of incidents", discussed and evaluated the problems encountered by the contractors' safety and the reasons for the lack of attention of contractors to safety issues from the technical and economic viewpoints, the training and control of waste and analysis.

In the electricity company, 66 urban development and improvement projects in Asalouyeh have been considered as a case study, the results of which show that the role of the employer in supervising the contractor in various stages of the contract until the end of the implementation is undeniable. Also, the results of the research show that culturalization is essential as a first step in preventing incidents. Obviously, due to the lack of proper culture and safety precautions, even there were events with the purchase of expensive protective equipment. In a study by Chen Yang et al. (2018), a dynamic model was proposed to simulate the chlorine process safety management system, which includes four modules for workers, management, rules (regulations) and equipment, as well as an integrated junction with the degree of corrosion that is comprehensively integrated. By introducing a series of scenarios with different inputs, it was determined that the proposed dynamic model can obtain the penetration pattern in the degree of equipment corrosion. The results of this study showed that work skills have a positive impact on system safety, while work stress mainly affects system safety during the second half of the life cycle. Yeo et al. (2018) conducted a study and theory results showed that a significant senior commitment in terms of human resources and the allocation of costs and suitability of the security manager was a key to the implementation of the safety management system. More than incident and incident costs, improved organizational frameworks and increased ratings of security voting were identified as the main benefits of implementing a safety management system. At the same time, factors such as adequate resources, close coordination and high returns were the key challenges for implementing an effective safety management system in Hong Kong. Dabirian and Safar (2016) in a research entitled "Dynamic Modeling of Building Safety Management System Based on Correctional Measures of the Site", using the dynamical approach of the system examined the structure of management safety management in construction workshops using the dynamical approach of the system. The results of this study show that severity and number of incidents as well as losses considerably decrease by using learning from safety inspection, investigation of incidents and corrective actions based on them and he safety performance of construction projects are improved. This study shows corrective actions as a useful policy to prevent incidents. Mariani et al. (2015) in a paper entitled, " "The dynamics of the system for modeling construction incidents", analyzed the occupational incidents in the construction project due to the probability of the variables affecting the events of construction projects, using the system dynamics method. The model of this research covers the process of occupational incidents and direct and indirect costs. Finally, this model creates OSH cost components that need 
to be controlled, as well as improves the supply chain of contracts and supervisors to improve the quality of employees. The results of the research show that the variables of work discipline and work hazards have the greatest impact on work incidents. Therefore, contractors in the construction project fully understand what work and which part of the supply chain should be improved. In addition, showing direct and indirect costs and occupational safety and health costs will provide the benefits of project budget control. Therefore, the project's budget for occupational safety and health costs will not be more than planning.

\section{Methodology}

\section{A. Case study features}

Sechahoon Bafgh Pelletizing Complex has been designed to produce 5 million tons of usable pellets in the iron and steel industry by direct reduction method to supply the required pellet in the steel industry. The site of the factory is located near Chaghart iron ore, located 13 kilometers north of Bafgh city, from Yazd province (115 kilometers from Yazd) in the central part of Iran with a longitude of 55028 and a latitude of 31042.

\section{B. FMEA risk assessment}

Risk assessment for the Sechahoon pelletizing plant was performed using the FMEA method. A total of 625 risks were identified in this series, of which 286 are high risk RPNs and need to be scrutinized. These risks were categorized according to their nature and consequences in five physical categories, exposure to dust, ergonomics, emergencies, and psychosocial risks, so that they can be reviewed in detail. Figure (1) shows the percentage of each sector's risks. As it turns out, the largest share is related to the physical risks as much as 95\%. For this reason, physical risks were divided into various mechanical, electrical, vibration, radiation, sound, heating, and fire sectors. The percentage of each of these risks in Figure 2 shows that the share of mechanical risks exceeds other risks, and then the electrical risks have a higher percentage of high-risk and high RPN risks that need to be addressed more often.

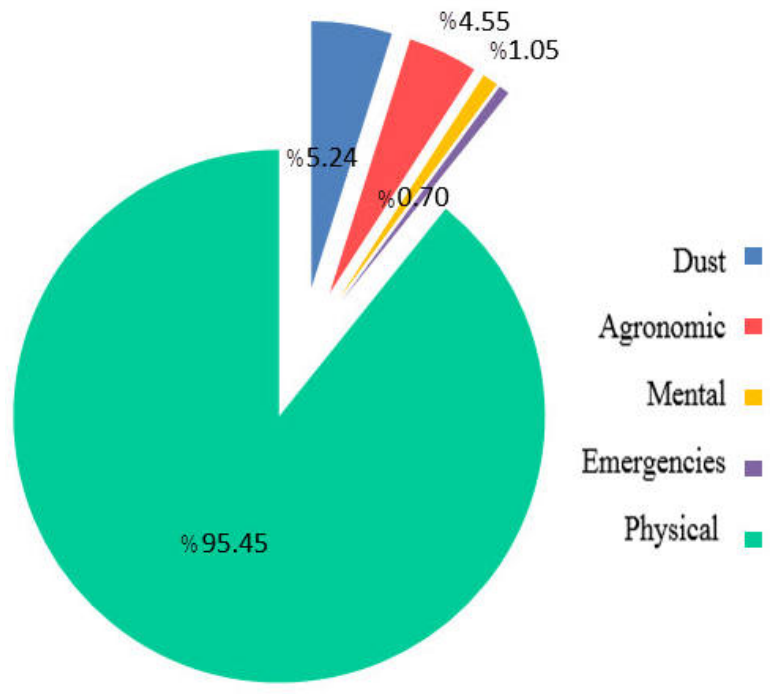

Figure 1. The extent of identified hazards by different sections 


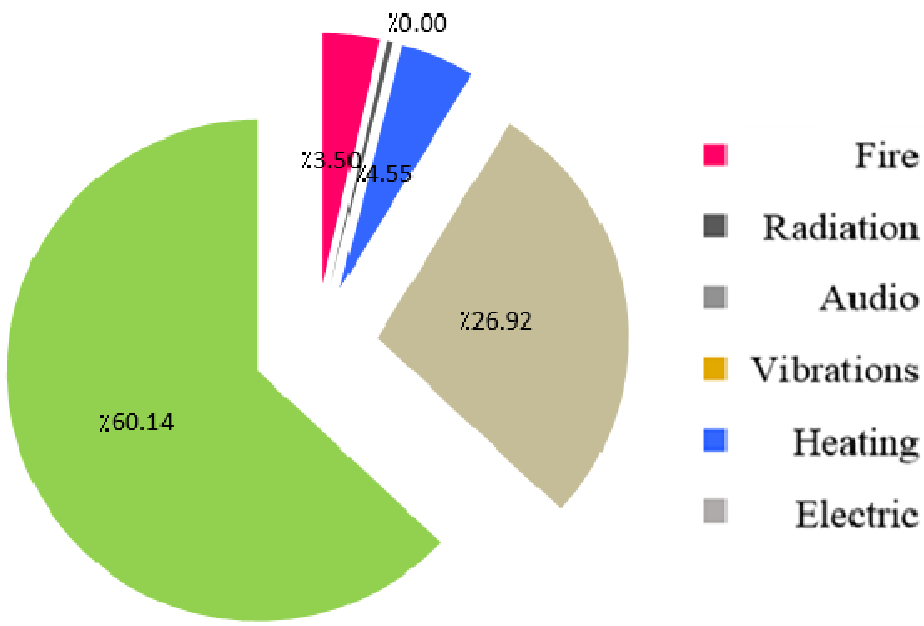

Figure 2. Physical hazards in different sections

\section{Results}

\section{A. Dynamic system model}

The number of incidents is one of the problems for each set. Moreover, the number of incidents as well as the lost days of the events should be examined to enhance the safety of existing processes and the factors affecting it, in addition to the risks in order to minimize their management and planning, their damage and the crisis. Figure 3 and 4 show the number of incidents and lost days, respectively. For this purpose, the model of dynamical system was created with information about different months in 2018 and its status was examined in 2019 and based on which different policies were proposed. After executing the simulated model, its validity needs to be measured.

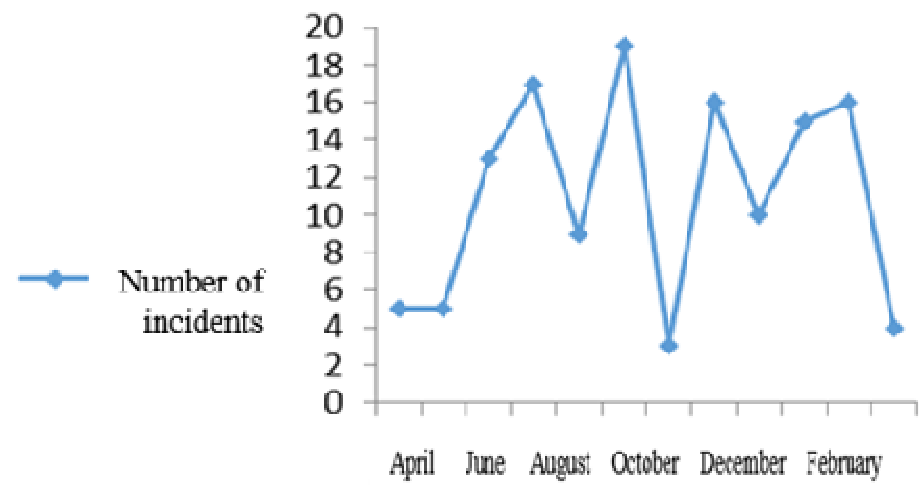

Figure 3. The number of incident in the pelletizing plant in 2018 in different months 


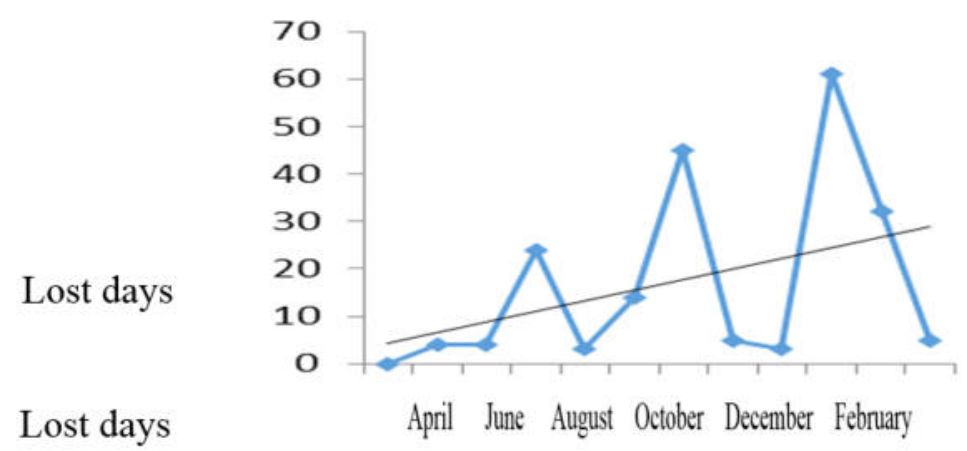

Figure 4. Number of lost days due to incident in 2018 by month

\section{B. Validation of the model}

After creating the flow chart of accumulation and simulation of the system and before using the model for analysis and scenario, one or more methods should be used to test the validity of the model. In this research, the validity of the model was investigated by several tests after simulation.

\section{Border adequacy test}

This test examines the important implications of the problem within the model. In this research, the proposed model has identified the key variables of the model after reviewing the literature. In addition, the necessity and importance of the variables mentioned by experts have also been studied. In response to the question of whether model behavior after the removal of border assumptions is a significant change, the results of the proposed model were studied after removing parts of the model and changing the boundary of the model. Figure 5 shows the graph of the effect of eliminating the "risk control measures" variable. Deleting this factor will reduce safety and increase incidents.

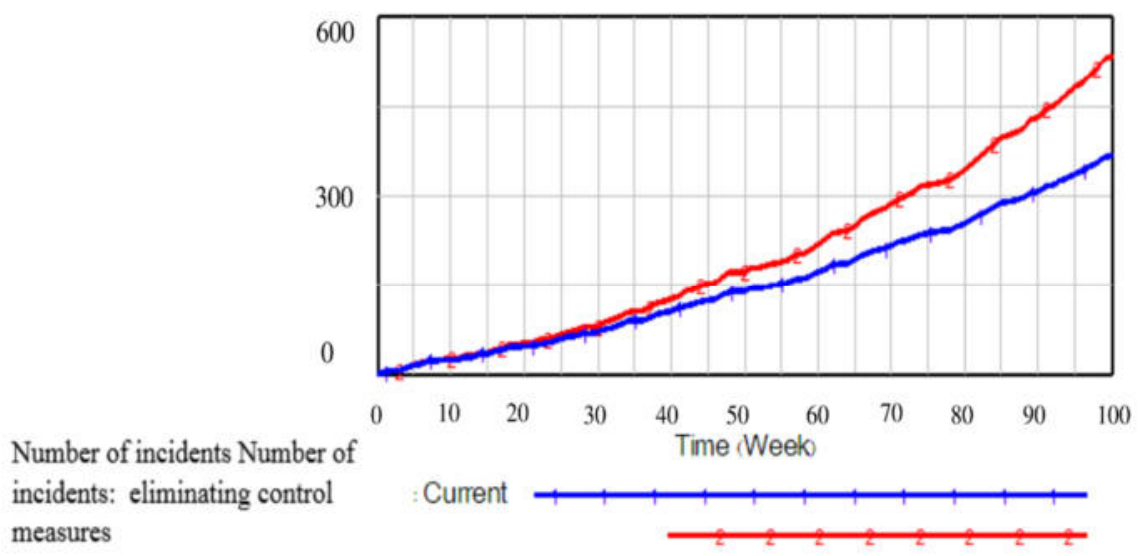

Figure 5. Border adequacy test

The graph compares the number of incidents related to the state of the safety control measures when these measures are eliminated. The number of events will be increased if these measures are removed. For other variables, this test can also be performed. 


\section{Structural Assessment Test}

The purpose of the structure test is to determine the model's structure matching with the descriptive knowledge associated with the system and to examine the logic of the decision rules in shaping the behavior of the variables and the correctness of the structure of the model equations. Since the model equations in the software environment have been written in this paper, the correctness of the structure of the model equations was verified by the software

(Figure 6).

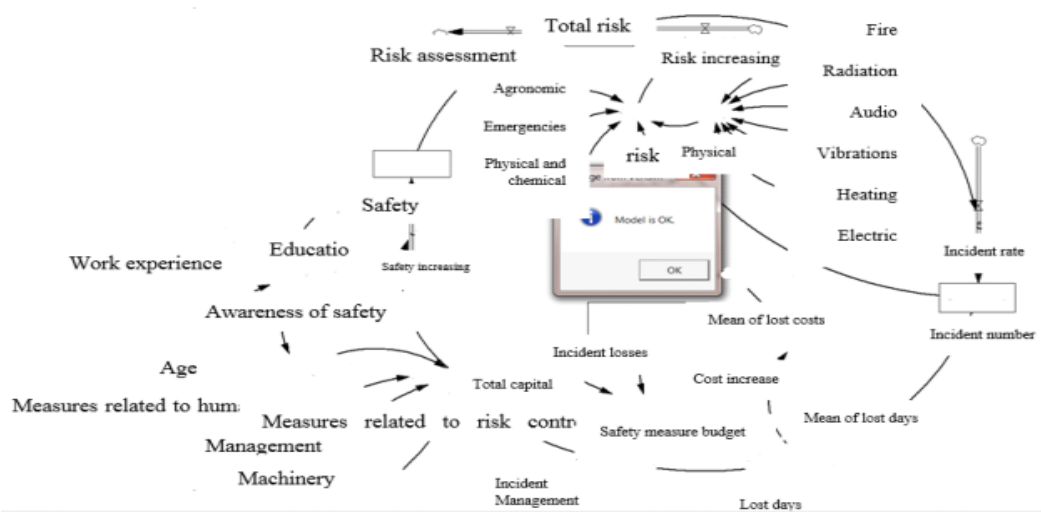

Figure 6. Structural assessment test

\section{E. Re-behavior test}

One of the most important tests available is the reproduction of behavior (compared with historical data). In this test it is determined which model variables can rebuild the amount of historical data. Figure 7 compares the outputs of the model with the actual data of the past. The matching will ensure modeling results and validate its validity in future prediction.

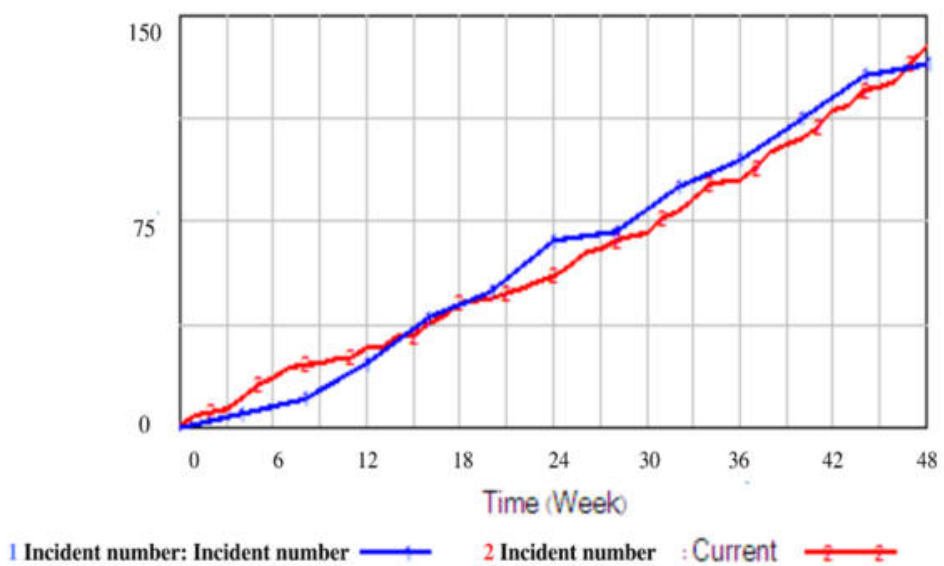

Figure 7. Re-behavior test 


\section{F. Boundary conditions test}

This test examines whether the model behaves appropriately when its inputs are in extreme conditions such as zero or infinity. In other words, in this test, the stability of the model is measured in extreme conditions. To examine this test, the variables "risk control measures", "safety" and "risk" were placed in their boundary condition, the results of which are shown in Figure 8.

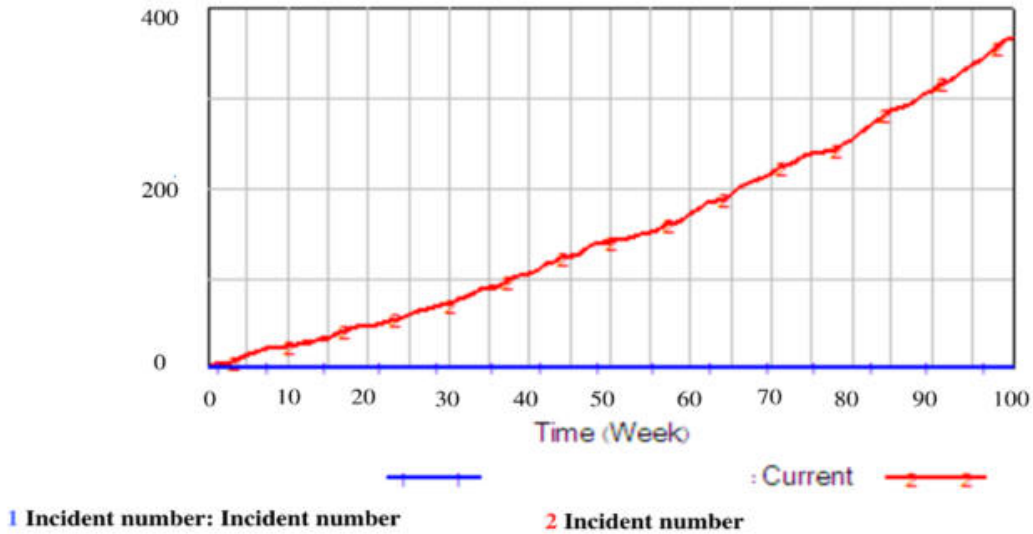

Figure 8. Boundary conditions test

\section{G. Sensitivity analysis}

In general, the purpose of the sensitivity analysis is to investigate whether changes in the parameters, boundaries, and time intervals result in significant changes in the numerical values, behavior, and policies observed or not. After simulating and observing the behavior of all components of the model in the desired time period, the change in the variables of the model and their impact analysis on the main variable is investigated. Table 1 shows the results of sensitivity analysis for various variables.

Table 1. Sensitivity analysis results

\begin{tabular}{|c|c|c|}
\hline Analytical results & Intervals & Variable \\
\hline $\begin{array}{l}\text { The number of incidents is susceptible and sensitivity } \\
\text { increases with time } \\
\text { Safety is high sensitivity and sensitivity increases with } \\
\text { time. }\end{array}$ & $60-15$ & Age \\
\hline $\begin{array}{l}\text { The number of incidents is susceptible and sensitivity } \\
\text { increases with time } \\
\text { Safety is high sensitivity and sensitivity increases with } \\
\text { time. }\end{array}$ & $30-0$ & Work Experience \\
\hline $\begin{array}{l}\text { The number of incidents is susceptible and sensitivity } \\
\text { increases with time } \\
\text { Safety is high sensitivity and sensitivity increases with } \\
\text { time. }\end{array}$ & $1-0$ & Education level \\
\hline $\begin{array}{l}\text { The number of incidents has a low sensitivity and } \\
\text { sensitivity does not increase with time. } \\
\text { Safety is sensitive and increases with time. }\end{array}$ & $1-0$ & $\begin{array}{l}\text { Measures related to human } \\
\text { resources }\end{array}$ \\
\hline
\end{tabular}




\begin{tabular}{|c|c|c|}
\hline $\begin{array}{l}\text { The number of incidents and safety is susceptible and } \\
\text { sensitivity increases with time. }\end{array}$ & $1-0$ & Safety Management Measures \\
\hline $\begin{array}{l}\text { The number of incidents has a low sensitivity and } \\
\text { sensitivity does not increase with time. }\end{array}$ & $1-0$ & $\begin{array}{l}\text { Safety measures related to } \\
\text { machinery }\end{array}$ \\
\hline $\begin{array}{l}\text { The number of incidents has a low sensitivity and } \\
\text { sensitivity does not increase with time. } \\
\text { Safety is sensitive and increases with time. }\end{array}$ & $1-0$ & $\begin{array}{l}\text { Safety measures related to the } \\
\text { environment }\end{array}$ \\
\hline $\begin{array}{l}\text { The number of incidents has a low sensitivity and } \\
\text { sensitivity does not increase with time. } \\
\text { Safety is sensitive and increases with time. }\end{array}$ & $5000000000-0$ & Safety Measures Budgeting \\
\hline $\begin{array}{l}\text { The number of incidents is not sensitive. } \\
\text { Safety has a low sensitivity and increases with time. }\end{array}$ & $1000000-0$ & Cost of one working day \\
\hline $\begin{array}{l}\text { The number of incidents is sensitive and increases with } \\
\text { time. } \\
\text { Safety is not sensitive. }\end{array}$ & $1-0$ & Heating risks \\
\hline $\begin{array}{l}\text { The number of incidents has high sensitivity and sensitivity } \\
\text { increases with time. } \\
\text { Safety is not sensitive. }\end{array}$ & $1-0$ & Mechanical risks \\
\hline $\begin{array}{l}\text { The number of incidents has high sensitivity and sensitivity } \\
\text { increases with time. } \\
\text { Safety is not sensitive. }\end{array}$ & $1-0$ & Electrical risks \\
\hline The number of incidents and safety is not sensitive & $1-0$ & Audio Risks \\
\hline The number of incidents and safety is not sensitive & $1-0$ & Vibration Risks \\
\hline $\begin{array}{l}\text { The number of incidents has a low sensitivity and increases } \\
\text { with time. } \\
\text { Safety is not sensitive. }\end{array}$ & $1-0$ & Fire and explosion risks \\
\hline The number of incidents and safety is not sensitive & $1-0$ & Radiation risks \\
\hline $\begin{array}{l}\text { The number of incidents has a low sensitivity and increases } \\
\text { with time. } \\
\text { Safety is not sensitive. }\end{array}$ & $1-0$ & $\begin{array}{l}\text { Dangers of exposure to dust } \\
\text { and toxic and chemical } \\
\text { substances }\end{array}$ \\
\hline $\begin{array}{l}\text { The number of incidents has a low sensitivity and increases } \\
\text { with time. } \\
\text { Safety is not sensitive. }\end{array}$ & $1-0$ & Ergonomic risks \\
\hline The number of incidents and safety is not sensitive & $1-0$ & Emergency risks \\
\hline $\begin{array}{l}\text { The number of incidents has a low sensitivity and increases } \\
\text { with time. } \\
\text { Safety is not sensitive. }\end{array}$ & $1-0$ & Mental risks \\
\hline
\end{tabular}

After analyzing the results of analyzing different variables and analyzing their impact on the main variable in the intervals, the effective and determinants of safety management and incidents were determined and the number of incidents is examined under the following scenarios:

\section{- Scenario of maintaining the current situation}


- Scenario of Human Resources Management

- Scenario of Technological improvement

- Scenario of Safety Management

- Scenario of Risk reduction

\section{H. Scenarios}

\section{Scenario of maintaining the current situation}

According to this scenario, the process of safety management is assumed to be in line with the past and without any change or adoption of a new policy. All variables are the same as those previously defined; the actual values of some parameters are as follows:

Management measures related to safety are equal to 0.5 (on a scale of 0 to 1), safety measures related to equipment 0.6 (scale 0 to 1 ), environmental safety measures 0.6 ( 0 to 1 scale). If such a process continues, the number of incidents will end at the end of the 100th week to 365 incidents. Figure 9 shows the process of the number of events in this scenario.

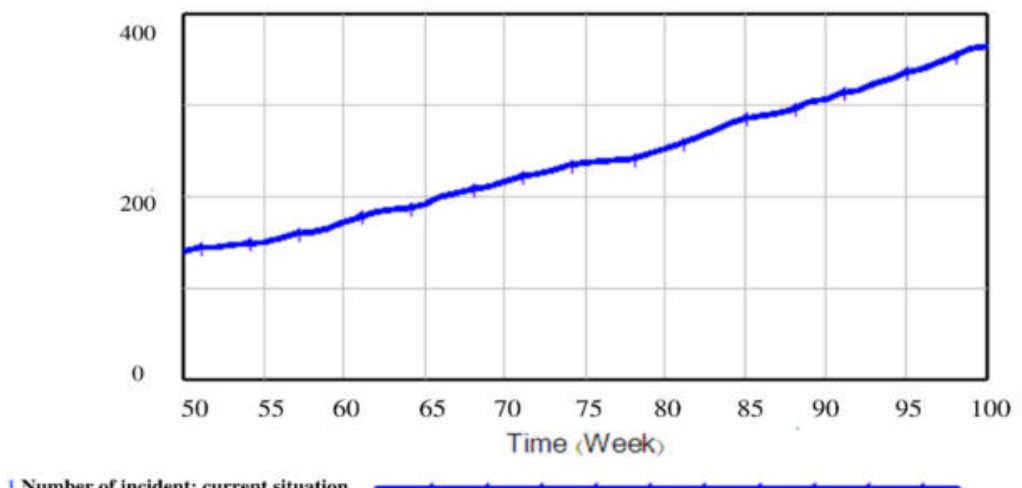

Figure 9. Scenario of maintaining the current situation

\section{Scenario of Human Resources Management}

The purpose of this scenario is to increase the safety of the human resource so that with its development increases the number of incidents. The human resource does not have the potential to fully cover occupational safety, but it can take a considerable amount of attention. Generally, the increase in human-resource risk control measures in this scenario began after about two months from the start and the use of human resources is considered with more education and more work experience. The number of incidents in this scenario with the values for the scenario of maintaining the current state is compared in Figure 10. 


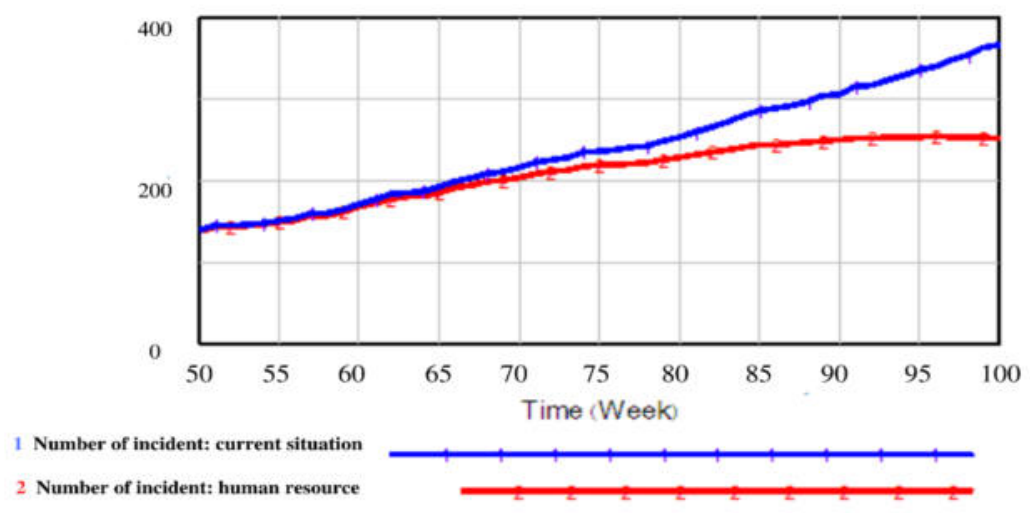

Figure 10. Comparison of two scenarios for maintaining the current status and human resource management In this scenario, the use of more advanced equipment and, subsequently, the detection and repair of machinerelated failures, their protection and, in general, the improvement of the operation of machinery, can be partly prevented from incidents.

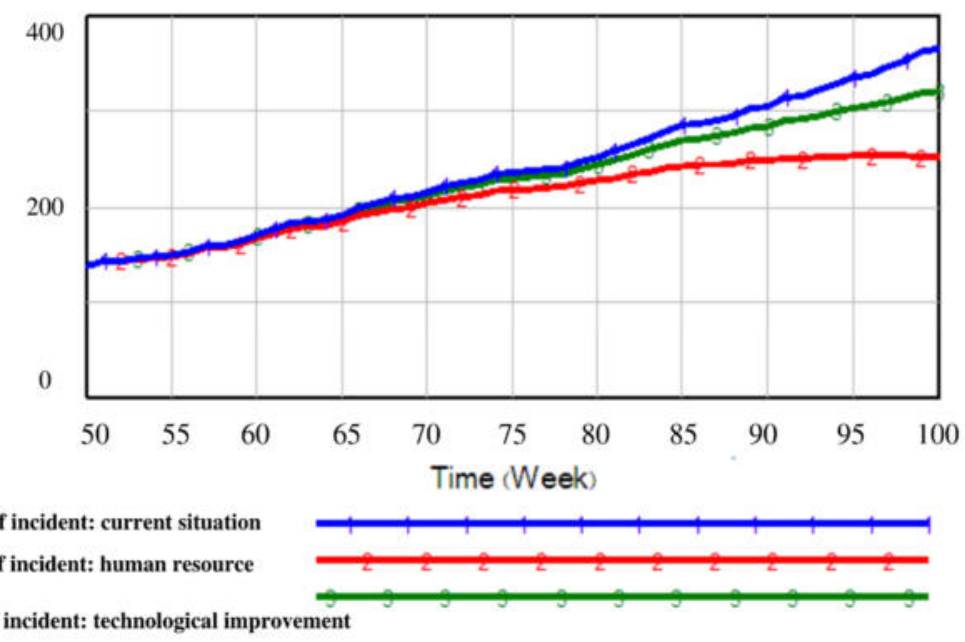

Figure 11. Scenario of Technological improvement

\section{Scenario of Safety Management}

The scenario shows that the number of incidents can be reduced by monitoring the performance of safety measures, monitoring the performance of safety measures, enforcing rules, creating guidelines for activities, and establishing monitoring, and development of safe spaces. The results of using this scenario are shown in Figure 12. As can be seen from the figure, the number of events will increase initially with the implementation of this scenario as in previous scenarios, and then it will decrease. 


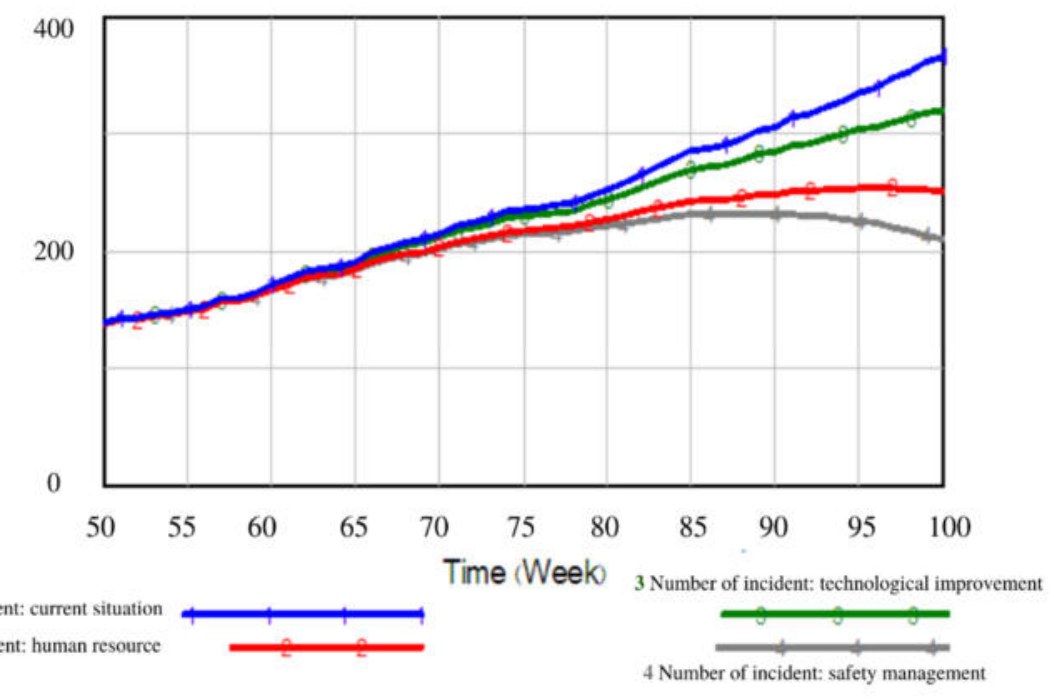

Figure 12. Scenario of Safety Management

\section{Scenario of Risk reduction}

In general, safety management scenarios are conceptually wide and diverse. However, the development of this scenario focuses on reducing the likelihood of incidents and increasing safety. The number of incidents will be reduced by creating these changes that have recently been made and reducing the risks, especially those that are more severe and referred to in the previous sections. Figure 13 shows the results of this scenario in comparison with previous scenarios. According to the figure, the greatest reduction in the number of incidents occurs with this scenario, and then it will increase.

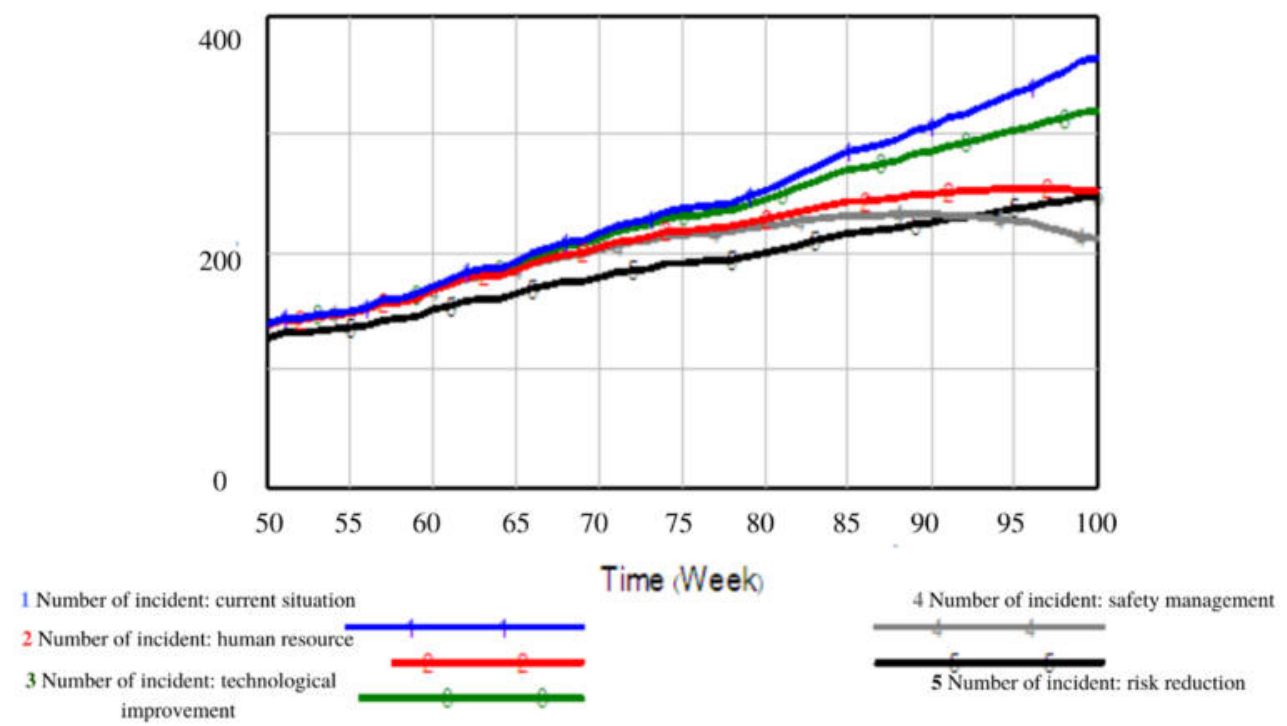

Figure 13. Risk reduction scenario 


\section{Scenario analysis}

The number of incidents in different scenarios is shown in Figure 13. In the risk reduction scenario, the reduction of incidents occurs earlier and is initially higher; however, increases again with time. This scenario can be effective in the short term. The scenario of technology improvement represents a slight upward trend, however, it is better than the scenario of maintaining the current situation and can improve reducing the number of incidents in the long run. Both of the human resource management and safety management scenarios are recommended as long-term effective methods. In either short or long term, two scenarios of risk reduction and safety management as two successive scenarios can be considered as the best scenarios in reducing the number of incidents. In the short term, the risk reduction scenario and in the long run the safety management scenario will have the best results in reducing the number of incidents.

\section{Conclusion}

In general, the results of this simulation indicated that reducing the number of incidents in the scenario of risk reduction is earlier, and is initially more than other policies. The scenario of risk reduction is the best scenario in the short term. The scenario of technology improvement shows an incremental growth trend, but the scenario of maintaining the current situation can better reduce the number of incidents. The scenario of human resource management and safety management scenario will initially be on an upward trend and will continue to decline. The scenario of human resource management and safety management are a good method to reduce disasters in the long run.

The safety management scenario is better than the scenario of human resources management. The best method to reduce the number of incidents is to successive use of two risk reduction and safety management scenarios. In the short term, the risk reduction scenario and in the long run the safety management scenario will have the best results in reducing the number of incidents.

With regard to the findings and limitations, it is recommended to evaluate the effect of other effective factors on the problem. The cost of running any of the scenarios can be an important issue for managers and its calculation can be helpful. Considering that electrical and mechanical risks have the highest share in increasing the probability of occurrence of incidents, the risk reduction scenario can reduce the number of incidents and be used as an optimal scenario in the short run. It is better to pay much attention to reform electrical and mechanical risks. The methods used to reform the risks will be effective when it reduces the number of incidents. Therefore, it is better to consider these methods more accurately.

\section{References}

Dousti, H. (2014). The Consequences of Work-related Accidents", Social, Economical, Scientific and Cultural Monthly Work and Society, No. 171.

Fathi Biranvand, A. 2017. Management of Accidents and Incidents in Building Projects with Dynamic System Approach", Third Annual Conference of Architectural, Urban and Urban Research, Urban Architecture and Architecture Institute of Safiran Rah and Mehrazi.

Khaje Amiri, A. (2014). The Relationship between the Establishment of Occupational Safety and Health Management System (OHSAS) and Disaster Reduction in the Components Industry Group 
(Case Study of Aria Industry Co.). Master's degree in Executive Management, Payanor University, West Tehran Center.

Kiajordi, A. 2011. Investigating factors affecting the incidence of accidents and incidents in the small and medium-sized workshops of Mazandaran province. Labor and Society, 138, 81-90.

Rauf, H., Moazzeni, M., \& Shafei, I. (2017). Safety Management of Contractors in Event Control", 4th Crisis Management Comprehensive Conference (HSE), Tehran, Permanent Secretariat of the Conference.

Souri, H., \& Mortazavi, M. 2011. Determination of High-Risk Behaviors of Employees in Relation to Health and Safety in an Automobile Complex in 2011. Journal of Arak University of Medical Sciences, 16 (3).

Taghinejad, A. (2017). Assessment of the Effect of Safety Management on Improving the Performance of Building Workshops and Providing Appropriate Strategies. Master's Degree in Civil Engineering, Engineering Tendency and Construction Management, Tabari Nonprofit Institutes of Higher Education.

Zomorodi, S., \& Nasrabadi, M. (2017). Performance Evaluation of Occupational Safety and Health Management Using the TQSM Model at Shahid Bahonar Harbor, Bandar Abbas, 4th International Conference on Environmental Planning and Management, Tehran, Faculty of Environment, University of Tehran. 